EPIDEMIOLOGY

\title{
Recent trends in diagnoses of HIV and other sexually transmitted infections in England and Wales among men who have sex with men
}

\author{
N Macdonald, S Dougan, C A McGarrigle, K Baster, B D Rice, B G Evans, K A Fenton
}

Sex Transm Infect 2004;80:492-497. doi: 10.1136/sti.2004.011197

See end of article for authors' affiliations

....................

Correspondence to: Neil Macdonald, HIV and Sexually Transmitted Infections Department, Health Protection Agency, Communicable Disease Surveillance Centre, 61 Colindale Avenue, London NW9 5EQ, UK; neil. macdonald@hpa.org.uk

Accepted for publication 2 September 2004

\begin{abstract}
Objectives: To examine trends in rates of diagnoses of HIV and other sexually transmitted infections (STIs) in men who have sex with men (MSM) in England and Wales between 1997 and 2002.

Methods: Estimates of the MSM population living in England and Wales, London and the rest of England and Wales were applied to surveillance data, providing rates of diagnoses of HIV and STIs and age group specific rates for HIV and uncomplicated gonorrhoea.

Results: Between 1997 and 2002, rates of diagnoses of HIV and acute STIs in MSM increased substantially. Rates in London were higher than elsewhere. Rises in acute STls were similar throughout England and Wales, except for uncomplicated gonorrhoea and infectious syphilis, with greater increases outside London. Rates of gonorrhoea diagnoses doubled between 1999 and 2001 (661/100 000, 1271/ $100000, p<0.001)$ in England and Wales followed by a slight decline to $1210 / 100000(p=0.03)$ in 2002 - primarily the result of a decline in diagnoses among men aged $25-34$ (1340/100 000, 1128/ $100000, p<0.001)$ and 35-44 $(924 / 100000,863 / 100000, p=0.03)$ in London. HIV was the third most common STI diagnosed in MSM in England and Wales and the second in London, with the highest rate (1286/100 000) found among men aged 35-44 in London in 2002.

Conclusions: Rates of diagnosis of HIV and other STIs have increased substantially among MSM in England and Wales. Increases show heterogeneity by infection, geography, and age over time. Rates in London were twice those seen elsewhere, with greatest changes over time. The observed changes reflect concomitant increases in high risk behaviour documented in behavioural surveillance survey programmes.
\end{abstract}

S ince the late 1990s, increases in sexually transmitted infections (STIs) and HIV diagnoses in men who have sex with men (MSM) have been reported from countries in Europe, North America, and Australia. ${ }^{1-5}$ These increases are generally attributed to changes in the sexual behaviour of MSM and, indeed, steady increases in high risk sexual behaviour have been reported over this period in countries that conduct behavioural surveillance. ${ }^{6-8}$ Explanations to account for this increase in risk taking are numerous, ${ }^{10}$ and in some instances controversial. An international comparison of treatment optimism conducted in 2000 in cities in Australia, Canada, France, and England using a standard scale found few MSM were optimistic in the light of new HIV drug therapies and suggested heterogeneity in men's responses to antiretroviral therapies in different countries. ${ }^{11}$ Researchers in London found similar rates of high risk sex reported among those optimistic about HIV treatments and those who were not, ${ }^{12}$ suggesting that treatment optimism alone would not account for the increase in risk taking. With no clear answers to explain these worsening trends it seems prudent to look in more detail at what HIV and STI surveillance can reveal.

This paper examines trends in diagnoses of HIV and other STIs in MSM since HAART became widely available in England and Wales. Data from a variety of surveillance and survey sources have been combined to present these trends within the context of the MSM population. This analysis is timely, not least because of the apparent rise in STI transmission in MSM, but also because of the availability of recent estimates of the population of MSM obtained from a national probability survey conducted in $2000^{13}$ and applied to the 2001 census. ${ }^{14}$

\section{METHODS}

In the United Kingdom, surveillance of STIs, and for the most part HIV, is based on reports from genitourinary medicine (GUM) clinics that provide free, open access, and confidential diagnostic services to the public. The surveillance methods have been previously described, ${ }^{1}$ and are summarised briefly.

\section{HIV infections}

Newly diagnosed HIV infections in England and Wales are reported to the Health Protection Agency, Communicable Disease Surveillance Centre (CDSC) by laboratories and clinicians. Voluntary confidential reporting methods are used to collect a range of epidemiological information including Soundex code,,$^{15}$ date of birth and gender, which permit the identification of duplicate reports of the same individual. This analysis includes all males reported with a first HIV diagnosis in England and Wales where the probable route of infection was sex between men. Data are from reports received by the end of 2003, censored at the end of 2002 to minimise the effects of delays in reporting and ascertainment of route of infection.

People living with diagnosed HIV infection and receiving care in England and Wales are reported annually to the Survey of Prevalent HIV Infections Diagnosed (SOPHID). ${ }^{16}$ Men aged 16-44, where the probable route of infection was sex between men and who were reported as living in England and Wales for each year between 1997 and 2002 were included.

Abbreviations: GUM, genitourinary medicine; HAART, highly active antiretroviral therapy; MSM, men who have sex with men; STls, sexually transmitted infections; UAl, unprotected anal intercourse 

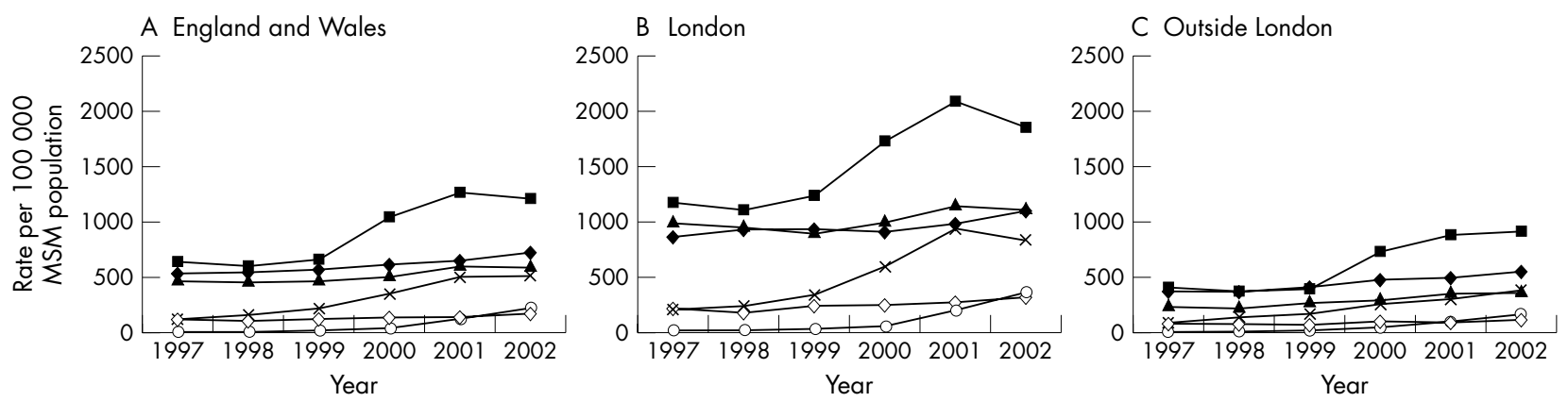

\begin{tabular}{ll|}
$\rightarrow-$ Uncomplicated gonorrhoea & $\rightarrow$ Genital chlamydial infection \\
$\rightarrow-$ Genital warts (first attack) & $\multimap$ Infectious syphillis (primary, secondary and early latent) \\
$\rightarrow-$ New HIV diagnosis & $\sim$ Genital herpes (first attack) \\
\hline
\end{tabular}

Figure 1 Trends in rates* of diagnoses of HIV† and other sexually transmitted infections $\ddagger$ in men who have sex with men. England and Wales 19972002 by year and region of diagnosis. (*Rates are based on estimated MSM populations aged 16-44 years of 277700 in England and Wales, 90000 in London and 187700 outside London. †Data source: Surveillance of new diagnoses of HIV infections. $¥$ Data source: KC60.)

\section{Other STIs}

Surveillance of other STIs in England and Wales is based on statutory quarterly aggregate statistical returns (KC60) from GUM clinics reported to CDSC and CDSC Wales. Male homosexual acquisition is reported for five acute conditions: uncomplicated gonorrhoea, infectious syphilis (primary, secondary, and early latent), genital chlamydia infection, genital warts (first attack), and genital herpes (first attack). Data on acute (male) homosexually acquired conditions were reviewed for the period 1997 to 2002. Information was available by region of diagnosis within England and for Wales for all five conditions. Age group at year of diagnosis is only reported for uncomplicated gonorrhoea in MSM.

\section{Estimating the populations of men}

The second UK National Survey of Sexual Attitudes and Lifestyles (Natsal 2000) ) $^{13}$ estimated the proportion of men, aged 16-44 years reporting sex with another man in the past 5 years to be $2.6 \%$ (95\% confidence interval 2.2 to 3.1 ) in Britain, 5.5\% (4.2 to 7.2) in Greater London, and 2.1\% (1.7 to $2.7)$ in the rest of Britain. These point prevalances were applied to the 2000 mid-year census estimate of males aged 16-44 years to obtain estimates of the numbers of MSM living in England and Wales as a whole, and separately for London and the rest of England and Wales, as well as for specific age groups based on the KC60 categories. Rates of diagnoses in heterosexual males, used as a comparison group for MSM, were estimated by subtracting diagnoses reported in MSM from those of men aged 16-44 and dividing these by adjusted population denominators (subtracting the MSM estimates from men aged 16-44).

\section{Statistical methods}

$\chi^{2}$ Tests and $\chi^{2}$ tests for trend were used to examine changes in the rates per 100000 MSM populations of diagnoses of HIV and other STIs between 1997 and 2002 using Epi-Info 6 (v.6.04d); 95\% confidence intervals around the rates were calculated using Stata 7 (StataCorp, 2001).

\section{RESULTS}

Between 1997 and 2002 the rate of diagnosis of all major acute STIs increased substantially among MSM in England and Wales (fig 1A). The biggest increases were in the rates of diagnoses of bacterial STIs, particularly between 1999 and 2001 , with a doubling in gonorrhoea (661/100 000 (95\% confidence interval $631 / 100000-692 / 100000)$ to $1271 /$ 100000 (1229 to 1313), p<0.001) and in chlamydia $(226 /$ 100000 (208 to 244$)$ to $504 / 100000(478$ to 552$), \mathrm{p}<0.001)$.
These increases were not sustained in 2002 with gonorrhoea decreasing to $1210 / 100000$ (1170 to 1252) ( $p=0.03)$ and chlamydia levelling off (524/100 000 (497 to 552), $\mathrm{p}=0.302$ ). Rates of diagnosis of syphilis rose from 7/100 000 (4 to 11 ) in 1997 to $225 / 100000$ (208 to 244$)$ in $2002(\mathrm{p}<0.001)$.

In comparison with the bacterial STIs, the increases in diagnosis rates of viral STIs between 1997 and 2002 were steadier, with genital warts rising from 536/100 000 (509 to $563)$ to $727 / 100000(696$ to 760$)(\mathrm{p}<0.001)$ and genital herpes from 121/100 000 ( 108 to 135) to $176 / 100000$ (161 to 192) $(\mathrm{p}<0.001)$. Rates of diagnoses of HIV infection increased from 478/100 000 (453 to 505) in 1997 to 601/ 100000 (572 to 630) in $2002(\mathrm{p}<0.001)$. Gonorrhoea remained the commonest STI diagnosed in MSM in England and Wales followed by genital warts, HIV and then chlamydia. Rates of diagnosis of syphilis overtook those of genital herpes in 2002 (fig 1A).

\section{London}

Diagnosis rates were markedly higher in London with gonorrhoea rising from a low of $1107 / 100000$ (1039 to 1178 ) in 1998, peaking at 2088/100 000 (1995 to 2184) in 2001 followed by a slight decrease to $1853 / 100000$ (1766 to 1945 ) in $2002(\mathrm{p}<0.001)$ (fig 1B). A similar pattern was seen for chlamydia, which also peaked in 2001 (938/100 000 (876 to 1003$)$ ) and decreased slightly to $843 / 100000$ (784 to 906) $(\mathrm{p}=0.024)$ the following year. Syphilis rose steeply from $50 /$ 100000 (36 to 67) in 2000 to $364 / 100000$ (326 to 406) in $2002(\mathrm{p}<0.001)$. HIV infection was usually the second most commonly diagnosed STI in MSM in London with an annual rate of diagnosis of around 950/100 000 (888 to 1017) between 1997 and 2000 rising to over 1100/100 000 (1038 to 1176$)$ in 2001 and $2002(p=0.001)$. Rates of diagnosis of genital warts were similar to HIV, increasing from 871/ 100000 ( 811 to 934$)$ in 1997 to $1101 / 100000$ (1034 to 1172 ) in $2002(\mathrm{p}<0.001)$. Herpes rose from $213 / 100000$ (184 to $246)$ to $313 / 100000(278$ to 352$)(\mathrm{p}<0.001)$ over this period.

\section{Outside London}

Although rates of diagnoses of HIV and other STIs were generally lower than those in London, the increases seen between 1997 and 2002 were of similar magnitude (fig 1C). The exceptions were gonorrhoea and syphilis, which exhibited larger increases outside London. Gonorrhoea rose steeply between 1999 and 2000 (385/100 000 (358 to 414) to 730/ $100000(692$ to 770$), \mathrm{p}<0.001)$ followed by a further increase to $879 / 100000$ (837 to 922$)$ in 2001 ( $p<0.01)$ and levelling off to $902 / 100000$ (859 to 946 ) in 2002. Syphilis 

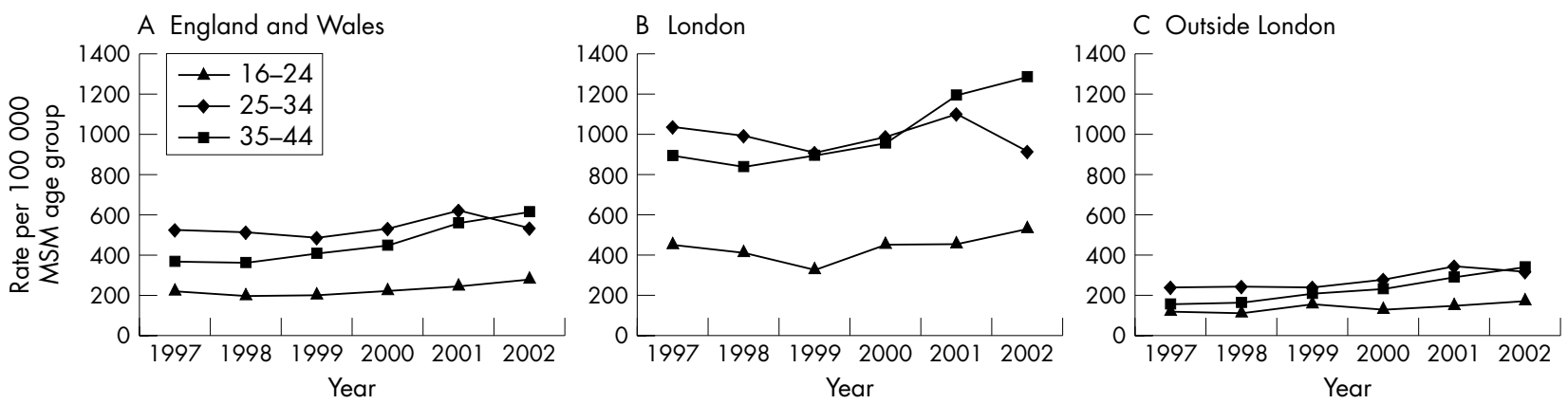

Figure 2 Trends in age group specific rates of diagnoses of HIV* in men who have sex with men. England and Wales 1997-2002 by year and region of diagnosis. ("Data source: Surveillance of new diagnoses of HIV infections.)

increased from 3/100 000 ( 1 to 7 ) in 1997 to $159 / 100000$ (141 to 178$)$ in $2002(\mathrm{p}<0.001)$.

\section{Age group specific trends HIV}

Between 1997 and 2002 in England and Wales, the rate of HIV diagnosis was highest in men aged 25-34, rising from $527 / 100000$ (486 to 570 ) in 1997 to $621 / 100000$ (577 to 668 ) in $2001(p=0.001)$ (fig 2A). Men aged 35-44 had the second highest incidence of diagnosis, rising steadily from 363/ 100000 (327 to 402) in 1998 to 613/100 000 (566 to 663) in $2002(\mathrm{p}<0.001)$ overtaking those aged $25-35$ that decreased to $534 / 100000$ (493 to 557) in that year. Men aged 16-24 generally had the lowest rate of HIV diagnosis. Nevertheless, diagnoses increased significantly from 200/100 000 (166 to 240 ) in 1998 to $283 / 100000$ (241 to 329) in $2002(\mathrm{p}=0.004)$. New HIV diagnosis rates were substantially higher for all age groups in London (fig 2B) compared with elsewhere (fig 2C). Between 2000 and 2001, rates in men aged 35-44 overtook those aged 25-34 in London. This crossover occurred a year later in the rest of England and Wales.

\section{Gonorrhoea}

Men aged 25-34 generally had the highest rate of diagnosis of gonorrhoea in England and Wales, closely followed by those aged 16-24. In contrast, men aged 35-44 had the lowest rate (fig 3A). Between 1997 and 1999 rates were fairly stable, but between 1999 and 2001 rates increased markedly in all age groups. This increase was maintained for men aged 16-24 in 2002 while rates decreased slightly in both the older age groups. Rates of diagnoses of gonorrhoea were higher for all age groups in London (fig 3B) compared to elsewhere (fig 3C). However, men aged 25-34 consistently had the highest rates in London. Outside London the highest incidence was seen in men aged 16-24, particularly from
1999 onwards. Between 2001 and 2002 rates in all age groups in London decreased, although this was only statistically significant for men aged 25-34 (2170/100 000 (2033 to 2314) to $1729 / 100000$ ( 1607 to 1858 ), $\mathrm{p}<0.001$ ) and men aged 35$44(1754 / 100000$ (1606 to 1911$)$ to $1525 / 100000$ (1388 to $1672), \mathrm{p}=0.028)$ and not for men aged 16-24 (1744/100 000 (1552 to 1953$)$ to $1540 / 100000$ (1360 to 1737$), p=0.138)$. Outside London the slight decrease in diagnoses in men aged 25-34 between 2001 and 2002 was not statistically significant $(865 / 100000$ (800 to 934$)$ to $784 / 100000$ (722 to 850$)$, $\mathrm{p}=0.082)$ and diagnoses in men aged 35-44 remained constant. The increase in men aged 16-24 continued in 2002 to $1243 / 100000$ (1138 to 1356).

\section{Comparing rates in MSM with heterosexual men}

Equivalent estimates of the rates of diagnoses of HIV and other STIs in heterosexual males were estimated in order to place the findings for MSM in context (table 1). This snapshot for England and Wales in 2002 indicates that rates were higher in MSM for all the STIs considered, ranging from 1.5 -fold for chlamydia (524/100 000 versus $341 / 100000)$ to over 50-fold for syphilis (225/100 000 versus 4/100 000).

\section{Prevalence of diagnosed HIV infection}

The prevalence of diagnosed HIV infection in MSM accessing care increased steadily from $2654 / 100000$ (2593 to 2715) in 1997 to $3706 / 100000$ (3635 to 3778) in 2002 (fig 4). The prevalence in MSM living in London and accessing care reached $7031 / 100000$ (6859 to 7207) in 2002 compared to $2111 / 100000$ (2046 to 2178) in men living elsewhere.

\section{DISCUSSION}

These results demonstrate the increases in rates of diagnosis of HIV and other STIs during the post-HAART era in MSM in England and Wales. The increases in rates of HIV and other
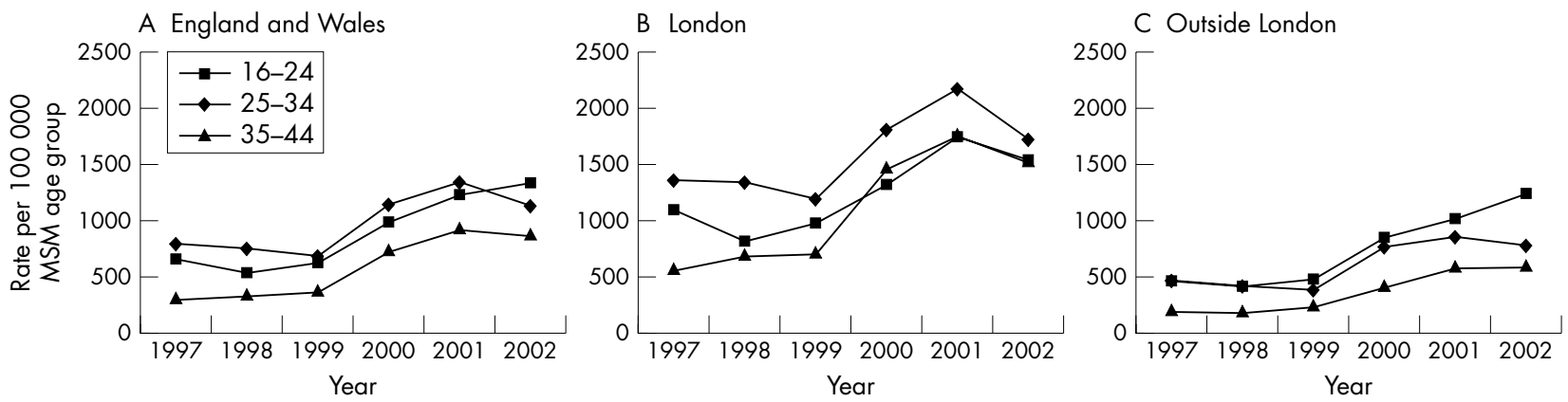

Figure 3 Trends in age group specific rates of diagnoses of uncomplicated gonorrhoea* in men who have sex with men in England and Wales $1997-$ 2002 by year and region of diagnosis. ('Data source: KC60.) 
Table 1 Rates* of diagnoses of HIV $\dagger$ and other STIs $\ddagger$ in men who have sex with men (MSM) and heterosexual men§ aged 16-44 in England and Wales in 2002

\begin{tabular}{lll}
\hline & MSM & Heterosexual men§ \\
\hline Uncomplicated gonorrhoea & $1210 / 10^{5}$ & $140 / 10^{5}$ \\
Genital warts (first attack) & $727 / 10^{5}$ & $337 / 10^{5}$ \\
New HIV diagnosis & $601 / 10^{5}$ & $13 / 10^{5}$ \\
Genital chlamydia (first attack) & $524 / 10^{5}$ & $341 / 10^{5}$ \\
Infectious syphilis (primary, secondary and early latent) & $225 / 10^{5}$ & $4 / 10^{5}$ \\
Genital herpes (first attack) & $176 / 10^{5}$ & $63 / 10^{5}$ \\
\hline *Rates are based on estimated populations of 277 700 MSM and 99999 900 heterosexual men aged 16-44 in \\
England and Wales in 2002). \\
†Data source: Surveillance of new diagnoses of HIV infections \\
†Data source: KC60 \\
\$Data on heterosexual males has been estimated by subtracting MSM from data for all males aged 16-44.
\end{tabular}

STIs in MSM in England and Wales have shown heterogeneity by type of infection, geographic location, and the age groups affected over time. Rates in London were twice those seen elsewhere and exhibited the greatest changes over time. There are some encouraging signs of a decrease in the rate of diagnosis of gonorrhoea between 2001 and 2002, but rates are still higher than in 1999 and to date, this decrease appears to be restricted to men aged over 25 in London.

The rise in rates of diagnoses of HIV and other STIs demonstrate increasing levels of sexual ill health in MSM and the burden placed on GUM services. Comparisons with heterosexual males reveal the extent of inequalities the sexual health of MSM. MSM in England and Wales in 2002 showed higher rates of diagnoses for all of the STIs investigated than heterosexual men, ranging from a doubling in rates of genital warts, herpes, and chlamydia, to over eight times for gonorrhoea and as high as 50 times for HIV and syphilis.

The heterogeneity in rates of diagnosis observed between STIs in MSM may relate to both differential transmission probabilities, levels of asymptomatic infection, and delays between infection and diagnosis. For some acute STIs the rate of diagnosis is likely to closely reflect the incidence of infection. This is particularly true for gonorrhoea where the onset of clinical symptoms usually occurs within a week of infection. ${ }^{17}$ Hence, rates of gonorrhoea are likely to be sensitive to changes in sexual behaviour, although treatment failure as a result of shifting patterns of antimicrobial resistance may also influence trends. Sentinel surveillance of antimicrobial resistance in England and Wales is provided by GRASP (Gonococcal Resistance to Antimicrobials Surveillance Programme). ${ }^{18}$ GRASP found that ciprofloxacin resistance $(\geqslant 1 \mathrm{mg} / \mathrm{l})$ in isolates from MSM increased from

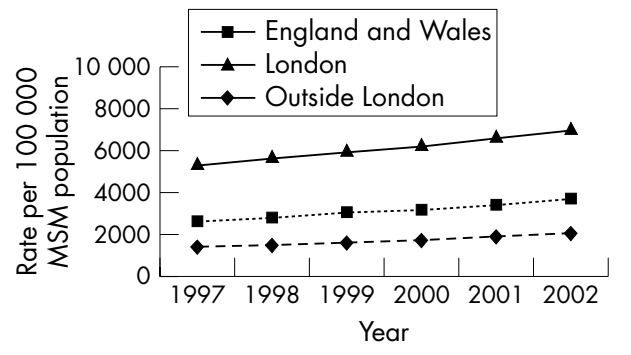

Figure 4 Trends in diagnosed $\mathrm{HIV}^{*}$ prevalencet in men who have sex with men, aged 16-44 accessing NHS services by year and area of residence. England and Wales 1997-2002. ('Data source: SOPHID. †Rates are based on estimated MSM populations aged 16-44 years of 277700 in England and Wales, 90000 in London and 187700 outside London.)
$0.8 \%$ in 2000 to $2.2 \%$ in 2001 with a further increase to $8.5 \%$ in 2002. ${ }^{19}$ Clinicians were notified of this decrease in susceptibility in May 2003; hence improved treatment is unlikely to account for the decrease in rates of diagnosis of gonorrhoea in MSM in London between 2001 and 2002.

Behavioural surveillance programmes, which monitor high risk sexual behaviour through sampling MSM attending commercial venues and GUM clinics in London, found the proportion of men reporting having engaged in unprotected anal intercourse (UAI) in the past year increased between 1997 and 2001. ${ }^{6}{ }^{12}$ However, no further increase in UAI was reported for 2002 and the proportion of men reporting UAI with partners of unknown or discordant HIV status decreased slightly from that reported for 2001. ${ }^{6}$ Such behaviour change may explain the decrease in rates of gonorrhoea observed in London. This apparent association between trends in high risk sexual behaviour and the rates of diagnosis of gonorrhoea in MSM requires further investigation, as surveillance of gonorrhoea may serve as a proxy for behavioural surveillance in areas where behavioural surveillance is lacking, such as in areas of England and Wales outside London. Further confirmation of this association would be provided through behavioural surveillance by asking men if they had been diagnosed with gonorrhoea.

The interpretation of trends in the incidence of diagnosis of HIV infection is more problematic. The delay between initial HIV infection and diagnosis may take years and consequently the rates described do not reflect the time or necessarily the place of infection. Nevertheless, between 1997 and 2002 there has been only a modest increase in the median age at diagnosis of HIV infection in MSM, and CD4 count at diagnosis has also remained fairly constant over this time. ${ }^{20}$ This suggests that the rates of diagnoses may indeed broadly reflect previous patterns of incidence. Application of a detuned assay to residual blood samples from MSM attending seven GUM clinics in London for syphilis serology found an annual incidence of around 3\% in these men with no statistically significant change between 1995 and 2001. ${ }^{21}$ That HIV was the third most commonly diagnosed STI in MSM in England and Wales (and the second in London) is surprising since the efficiency of transmission of HIV is believed to be far lower than other STIs..$^{22}$ This may in part reflect a higher probability of ascertainment for HIV, as undiagnosed infection eventually progresses to illness and death, compared to other STIs that may remain asymptomatic. Also, the duration of infectivity for HIV (lifelong) and the rising prevalence of MSM living with diagnosed HIV infection that we have reported, suggests that MSM will have a much higher probability of having sex with a man with HIV than any other major acute STI. Coupled with increases in both HIV risk behaviours and STIs that can enhance HIV transmission, 
the potential for future increases in HIV incidence is worrying.

Increasing numbers of MSM living with diagnosed HIV infection may also be contributing to the rising incidence of the other STIs. Enhanced syphilis surveillance has shown that MSM with diagnosed HIV infection feature disproportionately in the emerging syphilis outbreaks associated with metropolitan areas of England and Wales. ${ }^{23}$ In London almost half of the MSM diagnosed with syphilis were co-infected with HIV. A large cross sectional survey of MSM conducted in the United Kingdom in 2002 found diagnosed HIV positive men reported higher numbers of sexual partners and a greater likelihood of having been involved in HIV serodiscordant UAI compared to HIV negative and untested men. ${ }^{24}$ While UAI between diagnosed positive men may not be a concern for the onward transmission of HIV (although transmission of antiretroviral resistant virus between HIV positive men is), such circumstances can present an ideal environment for the transmission of other STIs.

Our study has some limitations. The rates described are probably more reliable for HIV than the other STIs described, since KC60 reports represent diagnoses within a quarter rather than individuals, but this effect might be balanced somewhat by under-reporting of male homosexual acquisition in KC60. Such under reporting will tend to overestimate the rates calculated for heterosexual males. Using MSM denominators of men aged 16-44 will tend to overestimate the rates of diagnosis for those STIs prevalent in men aged over 44; however, the age group specific rates are unaffected by this bias. Data from GUM clinics will underestimate the true rate of diagnosis in the population; however, this effect is likely to be small for MSM. A recent national survey of MSM found that although 79\% reported visiting a GP or local doctor in the past year, only $5 \%$ reported having a sexual health check up and $4 \%$ an HIV test, as a reason for their last visit to a GP surgery or local doctor. ${ }^{25}$ Open access for GUM means that patients may travel outside their area of residence to access services. This will tend to overestimate the rates for London (SOPHID recorded that $6 \%$ of HIV positive MSM seen for care in London in 2002 were resident outside London: B Rice, personal communication), but this effect is likely to be minimal for England and Wales as a whole. Although these limitations may result in a degree of error in the measurement of the rates described, this is likely to be consistent over time.

The trends presented may serve as a useful comparison with other countries where equivalent surveillance data and estimates of populations of MSM are available. A study of gonorrhoea in Denmark estimated the minimal yearly incidence between 1994 and 1999 to be 98/100 000 in MSM as a whole and 483/100 000 in MSM that were known to be HIV positive. ${ }^{26}$ Perhaps the small numbers of MSM reported in this study $(\mathrm{n}=266)$ dissuaded the authors from reporting annual estimates and trends. However, such estimates, in combination with other indicators (behavioural, attitudinal, and health promotion) could reveal the relative performance of HIV and STI prevention programmes targeted at MSM in different countries.

Many of the limitations of KC60 data for the surveillance of STIs in MSM described in this paper are likely to be overcome with the introduction of disaggregate reporting of STIs, which is currently under development in England and Wales. In the mean time, the data included in this analysis have been sufficient to describe the recent trends in HIV and STI diagnoses in MSM in England and Wales. It is perhaps too early to hope that the downward trend in the incidence of gonorrhoea in London will continue and extend to areas outside London. However, whatever the trajectory, the determinants of such temporal trends merit further investigation to determine the relative contributions of behavioural modifications (sexual or health service use), GUM access and service provision, and prevention interventions.

\section{ACKNOWLEDGEMENTS}

We gratefully acknowledge the contribution of the many people who report to, and support, the national HIV and STI surveillance systems, the Natsal Research team, and the Office for National Statistics. In addition to the many people who work in the HIV and Sexually Transmitted Infections Department at CDSC we thank Michael Toorie and Lee Hendry who provided ad hoc KC60 and SOPHID data for this paper.

\section{CONTRIBUTORS}

NM, SD, and CAM conceived the paper; NM and SD wrote the initial drafts; CAM provided behavioural data and made comments on drafts; BDR provided data from the SOPHID survey and commented on drafts; KB provided expert statistical support; $\mathrm{BE}$ and KF assisted in the provisions of data and commented on drafts.

\section{Authors' affiliations}

N Macdonald, S Dougan, C A McGarrigle, K Baster, B D Rice, B G Evans, K A Fenton, Health Protection Agency, Communicable Disease Surveillance Centre, 61 Colindale Avenue, London NW9 5EQ, UK

Conflict of interest: None.

\section{REFERENCES}

1 Health Protection Agency, SCIEH, ISD, National Public Health Service for Wales, CDSC Northern Ireland and the UASSG. Renewing the focus. HIV and other sexually transmitted infections in the United Kingdom in 2002. London: Health Protection Agency, November, 2003.

2 Dukers NH, Spaargaren J, Geskus RB, et al. HIV incidence on the increase among homosexual men attending an Amsterdam sexually transmitted disease clinic: using a novel approach for detecting recent infections. AIDS 2002; 16:F19-24.

3 Centres for Disease Control and Prevention. Increases in HIV diagnoses - 29 States, 1999-2002. MMWR Morb Wkly Rep, 2003;52;1145-8.

4 Remis RS, Alary M, Otis J, and the OMEGA Study Group, et al. Trends in HIV infection in the omega cohort of men who have sex with men (MSM) in Montreal, 1996-2001. Can J Infect Dis 2002;13(Suppl A):50A (abstract 320).

5 National Centre in HIV Epidemiology and Clinical Research (NCHECR). HIV/ AIDS, viral hepatitis and sexually transmissible infections in Australia: Annual Surveillance Report 2003, NCHECR, University of NSW, Sydney, 2003.

6 Dodds J, Mercey D. Sexual health survey of gay men-London 2002: summary of results. London: Royal Free and University College Medical School, September 2003.

7 Denning P, Nakashima AK, Wortley P. Increasing rates of unprotected anal intercourse among HIV-infected men who have sex with men in the Unites States. 13th International AIDS Conference, 9-14 July 2000, Durban, South Africa (Abstract no ThOrC714).

8 Van De Ven P, Prestage G, French J, et al. Increase in unprotected anal intercourse with casual partners among Sydney gay men in 1996-98. Aust N Z Public Health 1998;22:814-18.

9 Ostrow DE, Fox KJ, Chmiel JS, et al. Attitudes towards highly active antiretroviral therapy are associated with sexual risk taking among HIVinfected and uninfected homosexual men. AIDS, 2002 Mar 29, 16:775-80.

10 Stolte IG, Dukers NH, Geskus RB, et al. Homosexual men change to risky sex when perceiving less threat of HIV/AIDS since availability of highly active antiretroviral therapy: a longitudinal study. AIDS 2004;18:303-9.

11 City University and Royal Free and University College Medical School, London. International Collaboration on HIV Optimism. HIV treatments optimism among gay men. An international perspective. J Acquir Immune Defic Syndr 2003;32:545-50.

12 Elford J, Bolding G, Sherr L. High-risk sexual behaviour increases among London gay men between 1998 and 2001: what is the role of HIV optimism? AIDS 2002;16:1537-44

13 Johnson AM, Mercer CH, Erens B, et al. Sexual behaviour in Britain: partnerships, practises, and HIV-risk behaviours. Lancet 2001;358:1835-42.

14 Office for National Statistics. Census, April 2001. (Available at: www.statistics.gov.uk/census2001/ accessed 15 March 2003).

15 Mortimer JY, Salathiel JA. "Soundex" codes of surnames provide confidentiality and accuracy in a national HIV database. Commun Dis Rep 1995; 15:R183-6.

16 McHenry A, Macdonald N, Sinka K, et al. National assessment of prevalent diagnosed HIV Infections Commun Dis Public Health 2000;3:277-81.

17 Edward W. Gonococcal infection in the adult. Chapter 33. In: Holmes King K, eds. Sexually transmitted diseases, 3rd ed. New York. McGraw-Hill, 1999.

18 Paine TC, Fenton KA, Herring A, et al. GRASP: a new national sentinel surveillance initiative for monitoring gonococcal antimicrobial resistance in England and Wales. Sex Transm Infect 2001;77:398-401. 
19 Fenton KA, Ison C, Johnson AP, et al: GRASP collaboration. Ciprofloxacin resistance in Neisseria gonorrhoeae in England and Wales in 2002. Lancet 2000;361:1867-9.

20 PHLS Communicable Disease Surveillance Centre, ICH (London), SCEIH. HIV and AIDS in the United Kingdom 2001. London: An update November 2002.

21 Murphy G, Charlett A, Jordan LF, et al. HIV incidence appears constant in men who have sex with men despite widespread use of effective antiretroviral therapy. AIDS 2004; 18:265-72.

22 Anderson R. Transmission dynamics of sexually transmitted infections.

Chapter 3. In: Holmes King K, eds. Sexually transmitted diseases, 3rd ed. New York. McGraw-Hill, 1999.
23 Fenton KA. Sexual health and HIV positive individuals: emerging lessons from the recent outbreaks of infectious syphilis in England. Commun Dis Public Health 2002;5:4-6.

24 Hickson F, Weatherburn P, Reid D, et al. Out and about: findings from the United Kingdom Gay Men's Sex Survey 2002. Original Research Report. London: Sigma Research, 2003.

25 Keogh P, Weatherburn P, Henderson L, et al. Doctoring gay men: exploring the contribution of General Practice. Original Research Report. London: Sigma Research, 2004.

26 Johansen JD, Smith E. Gonorrhoea in Denmark: high incidence among HIVinfected men who have sex with men. Acta Derm Venereol 2002;82:365-8. 